\title{
A Protocol for Improved Measurement of Arterial Flow Rate in Preclinical Ultrasound
}

Authors

Affiliation

\section{A. Kenwright, A. J. W. Thomson, P. W. F. Hadoke, T. Anderson, C. M. Moran, G. A. Gray, P. R. Hoskins}

Edinburgh University, University-BHF Centre for Cardiovascular Science, Edinburgh, United Kingdom
Key words

- ultrasound Doppler

- technical aspects

- flow rate

- blood velocity

- preclinical

\section{Abstract}

$\nabla$

Purpose: To describe a protocol for the measurement of blood flow rate in small animals and to compare flow rate measurements against measurements made using a transit time flowmeter.

Materials and Methods: Measurements were made in rat and mice using a Visualsonics Vevo 770 scanner. The flow rate in carotid and femoral arteries was calculated from the time-average maximum velocity and vessel diameter. A correction factor was applied to correct for the overestimation of velocity arising from geometric spectral broadening. Invasive flow rate measurements were made using a Transonics system. Results: Measurements were achieved in rat carotid and femoral arteries and in mouse carotid arteries. Image quality in the mouse femoral artery was too poor to obtain diameter measurements. The applied correction factor

\section{Purpose}

$\nabla$

This paper relates to the measurement of flow rate in arteries in mice and rats. Flow rate in arteries is defined as the volume of blood passing the point of measurement per time unit, usually quoted in units of ml.min ${ }^{-1}$ or $\mathrm{ml}^{-\mathrm{s}^{-1}}$. Flow rate is a fundamental quantity which provides information related to the perfusion of downstream organs. Invasive methods have long been used to measure flow rate. This involves surgical exposure of the relevant artery and use of a probe hooked around the artery to measure flow rate. 2 main probes have been most commonly used to measure flow rate in this way: the electromagnetic flow (EMF) probe and the transit-time probe. The transit-time method has attained the status of a gold standard in preclinical flow measurement $[1,2]$. in practice was $0.71-0.77$. The diameter varied by $6-18 \%$ during the cardiac cycle. There was no overall difference in the flow rate measured using ultrasound and using transit-time flowmeters. The flow rates were comparable with those previously reported in the literature. There was wide variation in flow rates in the same artery in individual animals. Transit-time measurements were associated with changes of a factor of 10 during the typical 40 min measurement period, associated with probe movement, vessel spasm, vessel kinking and other effects.

Conclusion: A protocol for the measurement of flow rate in arteries in small animals has been described and successfully used in rat carotid and femoral arteries and in mouse carotid arteries. The availability of a noninvasive procedure for flow rate measurement avoids the problems with changes in flow associated with an invasive procedure.
In the last 15 years there has been mounting interest in the use of dedicated imaging systems (also called micro-imaging systems) for preclinical research. There are now available commercial preclinical imaging systems for ultrasound, MRI, CT, PET and fluorescence optical imaging [3]. MRI and ultrasound systems both have the capability of measuring flow rate in arteries noninvasively. VisualSonics (FUJIFILM VisualSonics, Toronto, ON, Canada) has produced a series of commercial ultrasound systems specifically designed for preclinical imaging with the first system launched in $2000[4,5]$. In ultrasound flow rate is estimated from the product of the measured mean velocity and cross-sectional area [6]. The accuracy of the measured flow rate is then determined by errors in the measurement of the mean velocity and area. The main work regarding the understanding of errors has been undertaken with respect to 
flow measured using clinical ultrasound systems [6,7]. Much of this understanding is translatable to preclinical ultrasound.

For the estimation of mean velocity, most of the literature uses the mean Doppler frequency. Under conditions of uniform insonation, where the entire cross-section of the vessel is within the sample volume, the mean Doppler frequency is an unbiased estimator of the mean velocity. However, requirements for high resolution mean that in practice this condition is rarely met. In practice the Doppler beam width in the elevation plane is typically much smaller than the vessel diameter. If the low velocities near the vessel wall are not insonated, there is overestimation of mean velocity [8]. The mean Doppler frequency is also highly sensitive to the location of the sample volume. Typically the operator will place the sample volume centrally in the vessel and try to align the axis of the vessel with the axis of the transducer. However, small displacements of the sample volume from the vessel center will result in changes to the mean Doppler frequency. For these reasons the measurement of mean Doppler frequency is not recommended and will not be considered further in this paper.

The mean velocity may also be estimated from the maximum Doppler frequency. Under conditions of fully developed flow, the time-averaged maximum velocity is twice the time-averaged mean velocity. This is true for steady (parabolic) flow, for quasisteady flow [8], and for pulsatile flow with reverse flow [9]. The measurement procedure then consists of measuring the timeaveraged maximum Doppler frequency shift and dividing this value by 2 . The maximum Doppler frequency shift is resistant to small movements of the sample volume, provided that the maximum Doppler frequency remains located at any point in the sample volume.

It has long been known that clinical ultrasound systems overestimate maximum velocity [10-13]. This is due to a phenomenon known as geometric spectral broadening [14]. The Doppler beam is generated from part of the linear array, which may be referred to as the 'Doppler aperture'. Essentially, clinical ultrasound systems assume that the width of the Doppler aperture is zero. The finite width of the aperture gives rise to an overestimation which is large, typically $0-60 \%$ for beam-target angles as used in clinical practice, increasing to over $100 \%$ with a beamtarget angle $>80^{\circ}$. Studies which have investigated this phenomenon have found good agreement between theory and experiment. Recently it has been shown that similar errors are present for maximum velocity measured using a preclinical system both for single element transducers [15] and array transducers [16]. The study by Kenwright et al. [16] demonstrated that a correction procedure could be used to reduce an angledependent error of up to $172 \%$ to $<25 \%$ for all angles.

The aim of the current paper is to report a protocol for the measurement of flow rate in mice and rats, and to compare flow rates measured using this protocol with those measured using a transit-time flow probe.

\section{Materials and Methods}

$\nabla$

Animal preparation

The rats were male Sprague Dawley aged 12 weeks and the mice were male C57 Black 6 aged 10 weeks. Animals were anesthetized using isoflurane ( $2 \%$ ), then placed in a supine position and the hair was removed from the skin overlying the carotid and femoral arteries using clippers and depilatory cream before measurements commenced. The animals' temperature was controlled using a heating pad. The animals were ventilated with $100 \%$ oxygen and isoflurane anesthesia. All animal experiments were approved by the University of Edinburgh preclinical ethical committee and by the UK Home Office.

\section{Ultrasound scanner}

Ultrasound scanning was performed using a Vevo 770 preclinical ultrasound scanner (FUJIFILM VisualSonics, Toronto, ON, Canada). This has a range of single-element high-frequency transducers with different frequencies and focal depths. In practice the RMV707B transducer (focal length: $12.7 \mathrm{~mm}$, frequency: $30 \mathrm{MHz}$ ) and the RMV704 transducer (focal length: $6 \mathrm{~mm}$, frequency) were used. The selected transducer was mounted on the Visualsonics integrated rail system incorporating a micromanipulator for precision adjustment of the probe position and orientation. Ultrasound was performed by one operator. Data were successfully acquired in the rat carotid and femoral and the mouse carotid. Attempts were made to image the mouse femoral but without success.

For diameter measurements the transducer was positioned to examine the artery in the cross-sectional plane and the transducer was orientated so that, as far as possible, the imaging plane was orthogonal to the vessel. The M-mode display was enabled so that diameter variation through the cardiac cycle could be seen. The image was frozen and on-board software was used to track the upper and lower wall trace to provide minimum and maximum diameter values ( $\boldsymbol{0}$ Fig. $\mathbf{1}$ ). The operator marked the beginning and end of the cardiac cycles to be included. Respiration was identified from the M-mode trace and avoided where possible. Typically 5-8 cardiac cycles could be obtained. The systolic diameter $\left(d_{s}\right)$ and diastolic diameter $\left(d_{d}\right)$ of each waveform were obtained. These were later averaged to produce a single value of $d_{d}$ and $d_{s}$ (see below).

For Doppler measurements the vessel was imaged in the longitudinal plane. Spectral Doppler was enabled and the sample volume placed centrally in the vessel to obtain spectral Doppler waveforms. The transducer angle and orientation were adjusted as necessary to obtain an angle less than $70^{\circ}$. The angle cursor was aligned parallel to the vessel wall. Doppler gain was adjusted until a few pixels were at maximum whiteness. This procedure was adopted to minimize errors arising from incorrect machine settings and probe placement to the greatest extent possible. This is discussed further in the Discussion section. The image was frozen and on-board software was used to trace the maximum Doppler frequency shift ( $\boldsymbol{0}$ Fig. 2). The operator marked the beginning and end of the cardiac cycles to be included. Respiration was identified from the Doppler trace as a reduction in the peak velocity and was avoided where possible. It is noted that maximum velocity may reduce due to other causes such as probe misalignment and natural variations in velocity so it was not always possible to identify definite periods of respiration. Typically 5-8 cardiac cycles could be obtained. The time-averaged maximum velocity $V_{\text {meas }}$ over all of the cardiac cycles was estimated by the on-board software.

The scanning time per animal was $20-30$ min involving optimization of the transducer position and alignment, adjustment of machine settings and trial recordings. The final measurements were based on at least 5-10 cardiac cycles of data. 


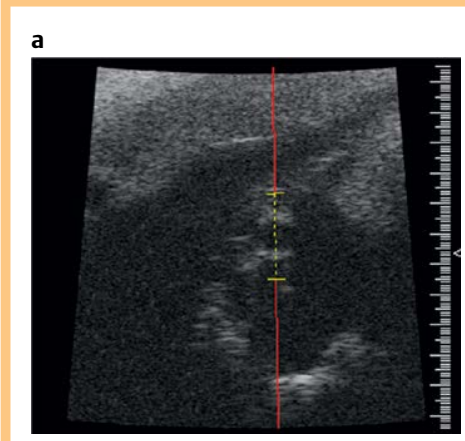

b

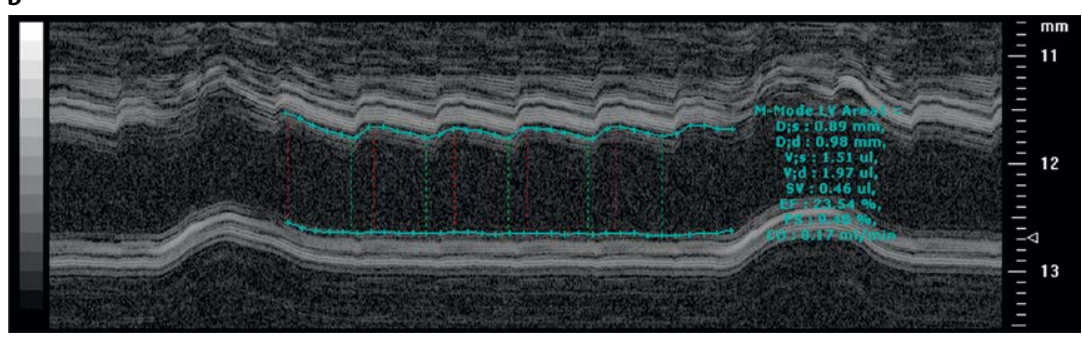

a

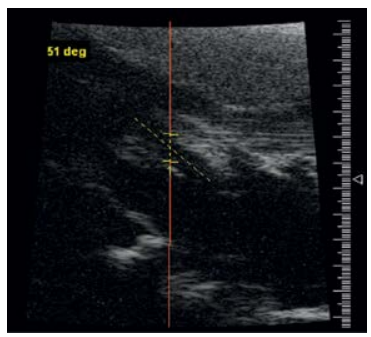

b

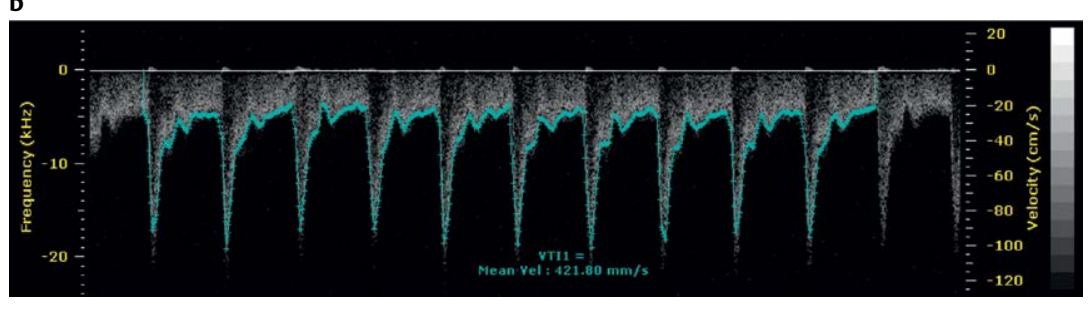

Fig. 1 a B-mode image of a rat carotid artery in cross section. $\mathbf{b} \mathrm{M}$-mode image showing automated measurement of diameter using the on-board software on the Visualsonics system.
Fig. 2 a B-mode image of a rat carotid artery shown longitudinally. b Doppler spectral trace showing automated maximum velocity estimation using the on-board software on the Visualsonics system.

\section{Transonics flowmeter}

Invasive flow measurements were taken using a transit-time flowmeter (TS410, Transonic Systems Inc., NY, USA). The measurement principle is that the speed of propagation of ultrasound between 2 fixed points is proportional to the flow rate. The probe contains an ultrasound emitter, a reflector and a receiver. The time of flight (transit time) between emitter and receiver is measured and the unit converts this to a flow measurement [17].

A skin incision was made at the site of the required artery and the artery was exposed by blunt dissection. The wound was irrigated using lignocaine to prevent the tissue from drying out and also to prevent vasospasm from occurring in response to manipulation of the artery. The flow was measured using probes which wrapped around the artery. A range of probes was available and the probe was chosen to suit the artery being scanned. In practice the MA1PRB (Transonic Systems Inc.) probe was used to scan the rat carotid and the MA0.7PSB (Transonic Systems Inc.) for the rat femoral and mouse carotid. Transonics flow measure- ments were performed by one operator. The probe was positioned around the exposed artery and gaps between the artery and probe were filled with Surgilube (E. Fougera \& Co. NY, USA), which has the same acoustic properties as blood ensuring that there was good fluid coupling to enable passage of ultrasound. The probe was carefully positioned with attempts to avoid kinking or trauma of the artery or surrounding tissues. There was variation in the measured flow associated with initial placement of the probe and probe movement. Stable traces were obtained after initial placement and flow only was measured during these stable portions. The probe was hand-held throughout the procedure with avoidance of movement during the recording phase.

\section{Scanning protocol}

For each artery measurements of blood flow were obtained using ultrasound and using the transit-time probe. Initially it was envisaged that simultaneous measurements of flow taken using the 2 methods would be the optimum protocol. However, in practice this was challenging as placement of the transit-time 
probe caused fluctuations in flow which took some time to settle down. Therefore, the use of non-simultaneous measurements was also investigated. The following series of measurements was undertaken:

Series 1 (rat-simultaneous). The carotid and femoral were exposed. Measurements of flow were taken using ultrasound on the carotid artery. The transit-time probe was positioned and measurements of the flow in the carotid artery were taken. Measurements of flow were taken using ultrasound in the femoral artery. The transit time probe was positioned and measurements of the flow in the femoral artery were taken. This was undertaken on 5 rats. The total procedure time was $40-50$ min for each animal.

Series 2 (rat-consecutive). Measurements of blood flow were taken on the carotid and femoral arteries using ultrasound. The carotid and femoral were exposed and measurements of flow were taken using the transit-time probe. This was undertaken on 7 rats. The total procedure time was $90 \mathrm{~min}$ for each animal. Series 3 (mouse-consecutive). Measurements of blood flow were taken on the carotid arteries using ultrasound. The carotid was exposed and measurements of flow were taken using the transit-time probe. This was undertaken on 6 mice. The total procedure time was $90 \mathrm{~min}$ for each animal.

\section{Correction of velocity measurements}

It was noted above that the maximum velocity measured using ultrasound is overestimated as a result of geometric spectral broadening. The equation for overestimation is shown below (Hoskins 2011):

$V_{\max }=V\left[1+\left(\frac{D}{2 L}\right) \tan \theta\right]$

where $V_{\max }$ is the maximum velocity estimated by the ultrasound system, $V$ is the true maximum velocity, $D$ is the Doppler aperture width, $L$ is the depth of the sample volume (equal to the focal length for these single-element transducers) and $\theta$ is the angle between the beam and the direction of motion. Rearranging this equation gives:

$V=\frac{V_{\max }}{\left[1+\left(\frac{D}{2 L}\right) \tan \theta\right]}$

For the maximum velocity $V_{\text {meas }}$ from the rat and mice, the corrected time-averaged maximum velocity $V_{\text {corr }}$ is given by equation 3:

$\mathrm{V}_{\text {corr }}=\mathrm{V}_{\text {meas }} \cdot \mathrm{CF}$

where the correction factor $C F$ is given by equation 4 :

$C F=\frac{1}{\left[1+\left(\frac{D}{2 L}\right) \tan \theta\right]}$

The values of $D$ and $L$ were $6 \mathrm{~mm}$ and $12.7 \mathrm{~mm}$ for the 707B transducer and $3 \mathrm{~mm}$ and $12.7 \mathrm{~mm}$ for the 704 transducer.

\section{Calculation of flow rate}

Diameter $d$ was estimated as the average of the minimum diastolic $d_{d}$ and maximum systolic $d_{s}$ diameters:

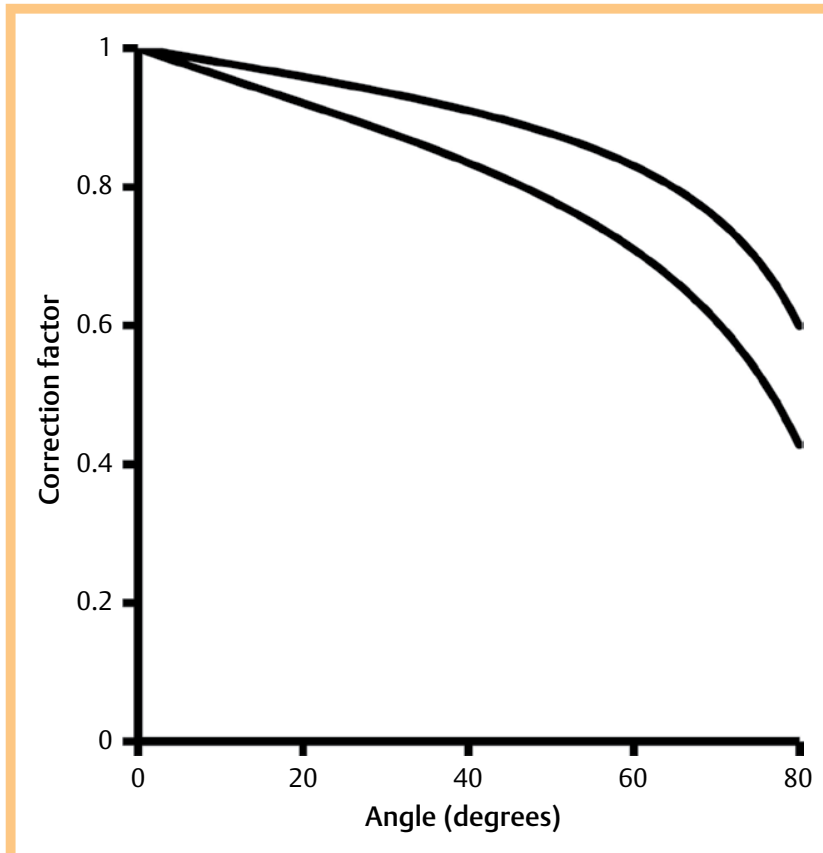

Fig. 3 Correction factor as a function of angle for the 704 and 707B transducers.

Table 1 Diameter measurements in all arteries; mean (sd).

\begin{tabular}{|llllc} 
& $\begin{array}{l}\text { Diastolic } \\
\text { diameter } \\
(\mathbf{m m})\end{array}$ & $\begin{array}{l}\text { Systolic } \\
\text { diameter } \\
\mathbf{( m m )}\end{array}$ & $\begin{array}{l}\text { Mean } \\
\text { diameter } \\
\mathbf{( m m )}\end{array}$ & $\begin{array}{l}\text { Systolic- } \\
\text { diastolic } \\
\text { variation (\%) }\end{array}$ \\
\hline Rat femoral & $0.52(0.2)$ & $0.55(0.2)$ & $0.537(0.2)$ & 6 \\
\hline Rat carotid & $0.94(0.2)$ & $1.06(0.2)$ & $1.00(0.2)$ & 13 \\
Mouse carotid & $0.44(0.06)$ & $0.51(0.06)$ & $0.47(0.06)$ & 18
\end{tabular}

$d=\frac{d_{s}+d_{d}}{2}$

Mean velocity $V_{m}$ was estimated as half the corrected time-averaged maximum velocity:

$V_{m}=\frac{V_{\text {corr }}}{2}$

Finally flow rate $Q$ was estimated as the product of the mean velocity and cross-sectional area.

$Q=V_{m} \frac{\pi d^{2}}{4}$

\section{Results}

\section{Correction factor}

- Fig. 3 shows the correction factor for the transducers. The impact of the correction factor increases with angle. This is expected since geometric spectral broadening also increases with angle. The measurements were taken at the shallowest angle achievable, which was between $50-60^{\circ}$ in all cases. This resulted in a correction factor of between $0.71-0.77$, i.e., the corrected velocity was between 23-29\% lower than reported by the on-board software. 

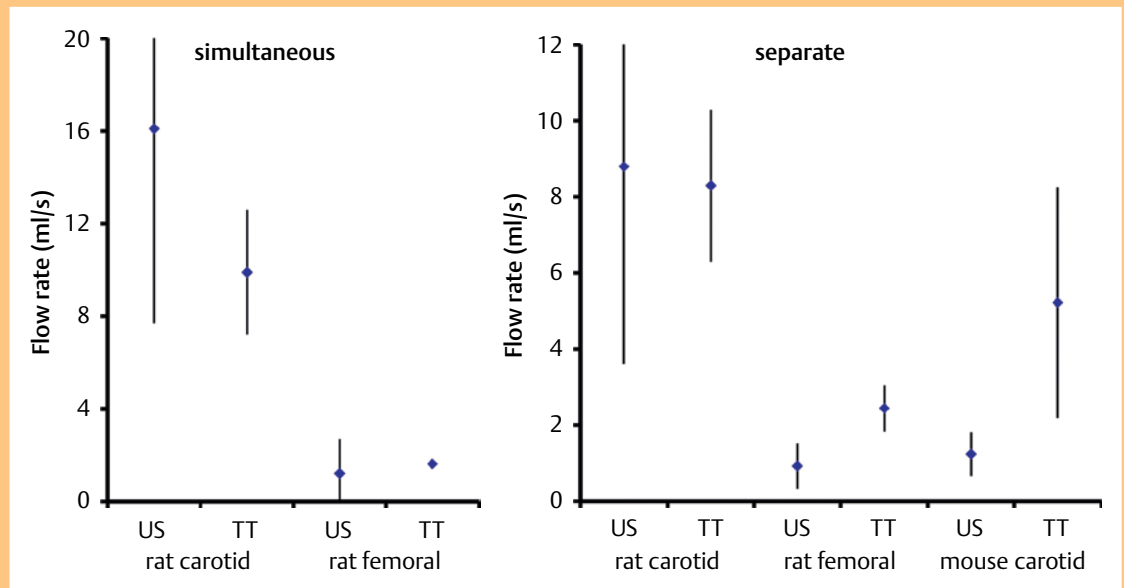

Fig. 4 Flow rates (mean, standard deviation) from the different arteries using ultrasound and using the transit-time flowmeter. Data are divided into a simultaneous measurement, $\mathbf{b}$ separate measurement.

\section{Diameter}

- Table 1 shows the measured diameter for each artery. The variation in diameter during the cardiac cycle was between $6-18 \%$.

\section{Flow rate}

- Fig. 4 shows the data from Series 1 (simultaneous measurements) and Series 2 and 3 (consecutive measurements). The highest flow rates were observed in the rat carotid artery in both the simultaneous and separate groups. Comparing ultrasound and transit-time measurements, the best agreement was achieved in the 'rat carotid separate' series ( $\bullet$ Fig. 5 ). The poorest agreement was seen in the mouse carotid where the transit time measurements were a factor of 4 higher than the ultrasound measurements.

- Table 2 shows the mean values of flow separated according to a number of groupings. Overall there was no statistically significant difference (paired t-test) between the flow measured using ultrasound and measured using the transit-time flowmeter for the 'separate', 'simultaneous' and 'rat carotid' groups. There was a difference $(\mathrm{p}<0.05)$ for the 'rat femoral' and 'mouse carotid' groups. The last column shows the data on the difference between the flow values in individual animals (median, range) which has a large range.

\section{Discussion \\ $\nabla$}

Errors in the estimation of the maximum velocity may arise due to a poor acquisition protocol. The operator generally attempts to reduce velocity errors by setting a low Doppler angle, setting the Doppler gain to an appropriate level (a few pixels at whiteout) and obtaining Doppler waveforms with a clearly defined outline. For the latter criterion, if there is misalignment of the beam and vessel, this will give rise to a Doppler trace with a noisy outline. Provided that due care and attention is taken, the remaining errors in maximum velocity estimation arise from geometric spectral broadening. A previous study has validated the method for correcting maximum velocity measurements in preclinical ultrasound where it was shown that errors arising from geometric spectral broadening reduced from up to $172 \%$ to $<25 \%[16]$.

The Transonic flow data were highly variable during measurements in a single session on a single animal ( $\bullet$ Fig. 6 ). In most cases after some probe manipulation, the flow stabilized allowing measurements to be taken. Movement of the probe had a

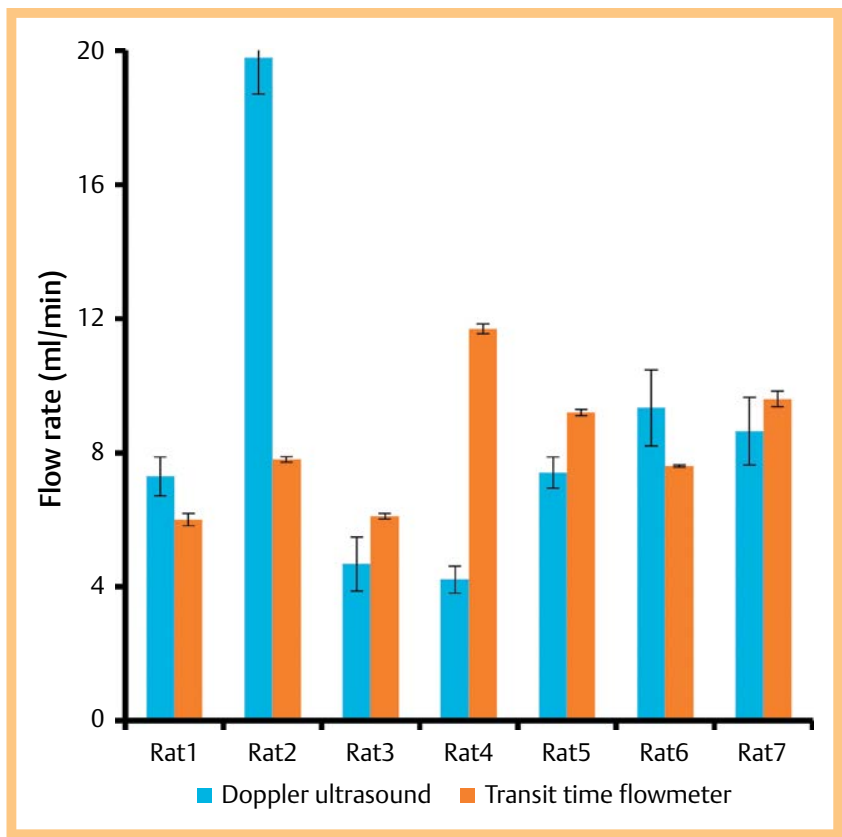

Fig. 5 Flow rate in individual rat carotid arteries using ultrasound and using the transit-time flowmeter.

Table 2 Flow rate (mean, sd) measured using ultrasound and using a transit-time flowmeter. The end column shows the difference in individual animals (median, range).

\begin{tabular}{|c|c|c|c|c|}
\hline & $\begin{array}{l}\text { Ultrasound } \\
\text { flow } \\
\left(\mathrm{ml} \cdot \mathrm{min}^{-1}\right)\end{array}$ & $\begin{array}{l}\text { Transit- } \\
\text { time flow } \\
\left(\mathrm{ml} \cdot \mathrm{min}^{-1}\right)\end{array}$ & p-value & Difference \% \\
\hline Separate (II, III) & $4.1(5.0)$ & $5.6(3.2)$ & 0.16 & $137(11-2900)$ \\
\hline Simultaneous (I) & $11.9(10.0)$ & $7.5(4.6)$ & 0.24 & $42(12-1450)$ \\
\hline Rat femoral & $1.00(0.81)$ & $2.2(0.6)$ & $0.04^{*}$ & $138(21-2112)$ \\
\hline Rat carotid & $11.8(7.4)$ & $9.0(2.3)$ & 0.23 & $28(11-178)$ \\
\hline Mouse carotid & $1.2(0.6)$ & $5.2(3.0)$ & $0.04 *$ & $171(136-2900)$ \\
\hline
\end{tabular}

significant effect on the recorded flow. Transit-time flow probes are reported as having a low position dependency on the measurement of flow (Drost 1978) so that the exact position of the vessel within the probe should not be a major factor. It is more likely that probe movement caused vessel kinking or spasm (though measures were taken to reduce spasm by irrigation with 


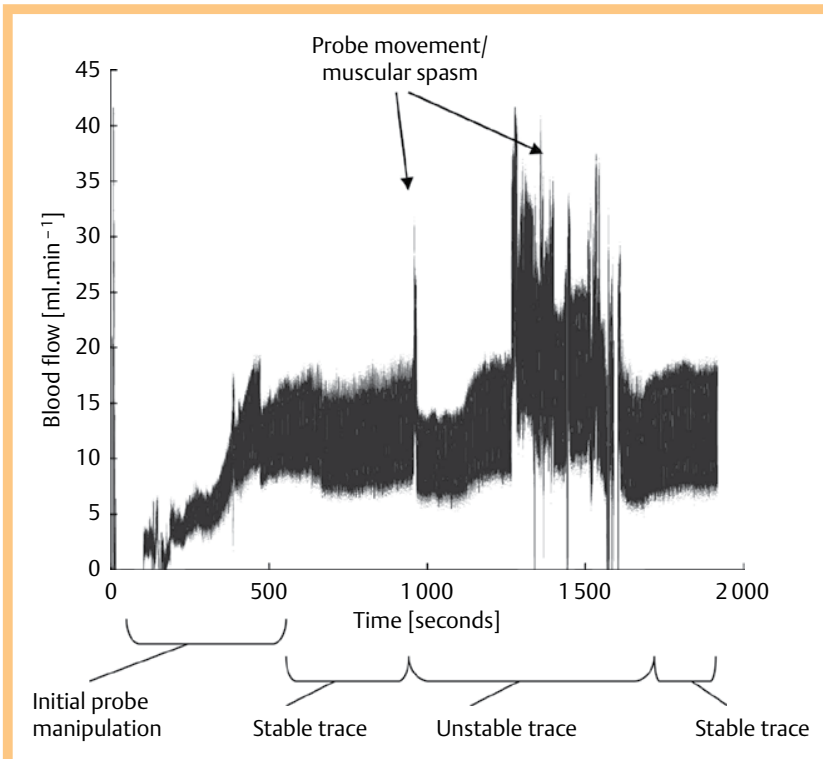

Fig. 6 Flow rate data in a single rat carotid artery over a period of 2000 s.

Table 3 Flow rate in comparison with the literature showing the range of values for each case. References are: rat carotid [18-21], rat femoral [19, 21-23], mouse carotid [24-28].

\begin{tabular}{|c|c|c|c|}
\hline & $\begin{array}{l}\text { Ultrasound flow } \\
\left(\mathrm{ml}^{\prime} \mathrm{min}^{-1}\right)\end{array}$ & $\begin{array}{l}\text { Transonic flow } \\
\left(\mathrm{ml}^{\prime} \mathrm{min}^{-1}\right)\end{array}$ & $\begin{array}{l}\text { Published } \\
\left(\mathrm{ml}^{\prime} \mathrm{min}^{-1}\right)\end{array}$ \\
\hline Rat carotid & $3.5-24$ & $6.0-12$ & $3.5-9$ \\
\hline Rat femoral & $1.5-3.1$ & $1.5-3.1$ & $0.8-3.7$ \\
\hline Mouse carotid & $0.3-1.7$ & $2.5-11$ & $0.4-2.1$ \\
\hline
\end{tabular}

lignocaine). There may also be changes in flow associated with changing metabolic demands (e.g., caused by temperature changes) and the anesthetic may have had an effect on blood flow.

A comparison of ultrasound and Transonic flow measurements demonstrated no overall difference when measurements were grouped according to 'separate' and 'simultaneous' protocols. When measurements were grouped according to artery and species, there was no significant difference for the rat carotid but there was a significant difference for the 2 smaller arteries (rat femoral and mouse carotid). These findings may simply reflect that both ultrasound and Transonic techniques are more reliable (and hence agree more) in larger superficial arteries.

A comparison with flow rates from the literature is shown in $\odot$ Table 3, with all data from I, II and III amalgamated. This is displayed in terms of range as the data from the literature are from different species of animal. For the rat femoral there is good agreement between the range of values found in this study and the literature. For the rat carotid there is also good agreement, noting that the maximum flow of $24 \mathrm{ml} / \mathrm{min}$ measured by ultrasound on one animal was much higher than that found in the literature. For the mouse carotid there is good agreement between the flow measured with ultrasound and that found in the literature. However, there is poor agreement between the Transonics flow measurements and the literature. The large range of values is noted in both published work and our study. Ideally a flow measurement method is needed which is noninvasive and reproducible. Measurement of flow using ultrasound does in principle offer this. In practice the ability to obtain highquality B-mode and spectral Doppler data varied between arteries and between animals. The best quality data were obtained in the rat carotid artery which is reasonably large and close to the surface, which are both conditions that help in terms of image quality. Obtaining data on the deeper rat femoral artery was more difficult. While it was possible to obtain good quality image data from the mouse carotid artery, it was not possible to obtain any good quality data in the mouse femoral artery. These technical issues will undoubtedly influence the accuracy of flow rate measured using ultrasound.

Future improvements in image quality and penetration depth will lead to better visualization of smaller and deeper arteries, allowing more accurate measurement of flow rate using ultrasound.

\section{Conclusion \\ $\nabla$}

A protocol has been reported for arterial flow measurement in preclinical ultrasound based on the measurement of diameter and maximum velocity and including correction for velocity overestimation due to geometric spectral broadening. The overall flow rate was comparable to that measured using a transittime approach. An ultrasound-based method is noninvasive and does not suffer from problems associated with surgical exposure of the artery. Ultrasound measurement of flow is likely to be reliable provided good quality data can be obtained from B-mode imaging and spectral Doppler.

\section{Acknowledgements}

This work was funded by the British Heart Foundation.

\section{References}

1 D'Almeida MS, Cailmail S, Lebrec D. Validation of transit-time ultrasound flow probes to directly measure portal blood flow in conscious rats. Am J Physiol - Heart Circ Physiol 1996; 271: H2701-H2709

2 Beldi G, Bosshard A, Hess OM et al. Transit time flow measurement: experimental validation and comparison of three different systems. Ann Thorac Surg 2000; 70: 212-217

3 Kagadis GC, Loudos G, Katsanos $K$ et al. In vivo small animal imaging: Current status and future prospects. Med Phys 2010; 37: 6421-6442

4 Foster FS, Zhang MY, Zhou YQ et al. A new ultrasound instrument for in vivo microimaging of mice. Ultrasound Med Biol 2002; 28: 1165-1172

5 Foster FS, Mehi J, Lukacs $M$ et al. A new 15-50 MHz array-based micro-ultrasound scanner for preclinical imaging. Ultrasound Med Biol 2009; 35: 1700-1708

6 Hoskins PR. Measurement of blood velocity, volumetric flow and wall shear rate. Ultrasound 2011; 19: 120-129

7 Evans DH, McDicken WN. Doppler ultrasound: physics, instrumentation and signal processing. Chichester: Wiley; 2000

8 Evans $\mathrm{DH}$. On the measurement of the mean velocity of blood flow over the cardiac cycle using Doppler ultrasound. Ultrasound Med Biol 1985; 11: 735-741

9 Li SF, Hoskins PR, Anderson T et al. Measurement of mean velocity during pulsatile flow using time averaged maximum frequency of Doppler ultrasound waveforms. Ultrasound Med Biol 1993; 19: 105-113

10 Daigle RJ, Stavros AT, Lee RM. Overestimation of velocity and frequency values by multi-element linear array Dopplers. J Vasc Technol 1990; 14: 206-213

11 Hoskins PR, Li SL, McDicken WN. Velocity estimation using duplex scanners. Ultrasound Med Biol 1991; 17: 195-199

12 Thrush AJ, Evans DH. Intrinsic spectral broadening: a potential cause of misdiagnosis of carotid artery disease. J Vasc Invest 1995; 1: 187-192

13 Hoskins PR. Accuracy of maximum velocity estimates made using Doppler ultrasound systems. Br J Radiol 1996; 69: 172-177 
14 Newhouse VL, Furgason ES, Johnson GF et al. The dependence of ultrasound Doppler bandwidth on beam geometry. IEEE Trans Sonics Ultrasonics 1980; 27: 50-59

15 Yang X, Anderson T, Moran CM et al. Assessment of spectral Doppler in preclinical ultrasound using a small-size rotating phantom. Ultrasound Med Biol 2013; 39: 1491-1499

16 Kenwright DA, Anderson T, Moran CM et al. Assessment of spectral Doppler for an array-based preclinical ultrasound scanner using a rotating phantom. Ultrasound Med Biol 2015

17 Drost $C J$. Vessel diameter-independent volume flow measurements using ultrasound. Proc San Diego Bioml Symp 1978; 17: 299-302

18 Kubo-Inoue M, Egashira K, Usui $M$ et al. Long-term inhibition of nitric oxide synthesis increases arterial thrombogenecity in rat carotid artery. Am J Physiol Heart Circ Physiol 2002; 282: H1478-H1484

19 Basu P, Sen U, Tyagi $N$ et al. Blood flow interplays with elastin: collagen and MMP: TIMP ratios to maintain healthy vascular structure and function. Vasc Health Risk Manag 2010; 15: 215-228

20 Mondy JS, Lindner V, Miyashiro JK et al. Platelet-derived growth factor ligand and receptor expression in response to altered blood flow in vivo. Circ Res 1997; 81: 320-327

21 Hoffman A, Grossman E, Ohman KP et al. Endothelin induces an initial increase in cardiac output associated with selective vasodilation in rats. Life Sci 1989; 45: 249-255
22 Rickard RF, Wilson J, Hudson DA. Characterization of a rodent model for the study of arterial microanastomoses with size discrepancy (smallto-large). Lab Anim 2009; 43: 350-356

23 Lundberg G, Luo F, Blegen $\mathrm{H}$ et al. A rat model for severe limb ischemia at rest. Eur Surg Res 2003; 35: 430-438

24 Ota R, Kurihara C, Tsou TL et al. Roles of matrix metalloproteinases in flow-induced outward vascular remodeling. J Cereb Blood Flow Metab 2009; 29: 1547-1458

25 Wilson KM, Lynch CM, Faraci FM et al. Effect of mechanical ventilation on carotid artery thrombosis induced by photochemical injury in mice. J Thromb Haemost 2003; 1: 2669-2674

26 Dörffler-Melly J, de Kruif M, Schwarte LA et al. Functional thrombomodulin deficiency causes enhanced thrombus growth in a murine model of carotid artery thrombosis. Basic Res Cardiol 2003; 98: 347-352

27 Bryant SR, Bjercke RJ, Erichsen DA et al. Vascular remodeling in response to altered blood flow is mediated by fibroblast growth factor-2. Circ Res 1999; 84: 323-328

28 Rudic RD, Bucci $M$, Fulton $D$ et al. Temporal events underlying arterial remodeling after chronic flow reduction in mice: correlation of structural changes with a deficit in basal nitric oxide synthesis. Circ Res 2000; 86: 1160-1166 